\title{
Comparative genomics between fly, mouse, and cattle identifies genes associated with sire conception rate
}

\author{
G. Li, ${ }^{* 1}$ F. Peñagaricano, $\dagger^{1}$ K. A. Weigel, $\neq$ Y. Zhang, ${ }^{*}$ G. Rosa, $\dagger$ and H. Khatib $\dagger^{2}$ \\ ${ }^{*}$ College of Animal Science and Technology, China Agricultural University, Beijing, 100193, China \\ †Department of Animal Sciences, and \\ ‡Department of Dairy Science, University of Wisconsin-Madison, Madison 53706
}

\section{ABSTRACT}

The decline in reproductive performance in cattle is of major concern to farmers and the dairy industry worldwide. Most fertility studies in cattle have focused on fertility of the cow, whereas the genetics of male fertility have not been thoroughly investigated. The present study hypothesizes that the high conservation of spermatogenesis genes from fly to human implies important roles of these genes in male fertility in cattle. To test this hypothesis, we performed an association analysis between highly conserved spermatogenesis genes and sire conception rate (SCR) in US Holsteins as a measure of bull fertility. Sequencing analysis revealed 24 single nucleotide polymorphisms (SNP) in 9 genes in the bull population using the pooled DNA sequencing approach. Five SNP previously identified in 5 genes from the POU1F1 pathway were also included in this study because they have shown significant associations with female and male fertility traits. Overall, 29 SNP located in 14 candidate genes were tested for association with sire conception rate in a population of 1,988 bulls. Three SNP located in MAP1B and 1 SNP in PPP1R11 showed significant associations with SCR. For the POU1F1 pathway, single gene analysis revealed significant associations of POU1F1 and STAT5A with $\mathrm{SCR}$. Analysis of genotypic interactions between adjacent genes in the pathway revealed significant associations of STAT5A and UTMP genotypic combinations with SCR. The most significant spermatogenesis gene, $M A P 1 B$, was found to be associated with fertilization and blastocyst rates. Thus, the association of these genes with bull fertility testifies to the usefulness of the comparative genomics approach in selecting candidate male fertility genes.

Key words: spermatogenesis, sire conception rate, cow fertility

Received April 2, 2012.

Accepted July 12, 2012.

${ }^{1}$ The first 2 authors contributed equally to this study.

${ }^{2}$ Corresponding author: hkhatib@wisc.edu

\section{INTRODUCTION}

Fertility is a complex trait that comprises multiple layers and developmental stages such as combining sperm and egg to make a zygote, compaction of embryo cells to form a morula, establishment of the blastocyst, attachment of the embryo to the uterus, and fetal development (Amann and DeJarnette, 2012). This complexity makes accurate prediction of successful pregnancy difficult; aberrant development of a sperm, oocyte, embryo, or fetus would lead to conception failure. The conception rate in dairy cattle is about $40 \%$, and only $50 \%$ of the fertilized eggs produce viable embryos (Santos et al., 2004). The decline in reproductive performance in cattle over the past few decades (Dobson et al., 2007) has been ascribed primarily to fertilization failure and early embryonic loss (Santos et al., 2004). Although many factors are involved in infertility, previous studies have shown that the genetic makeup of an individual plays crucial roles in embryonic development and reproductive success (Weigel, 2006; Shook, 2006).

Although males and females each contribute half of their genetic material to the new zygote and both are necessary for embryo development, it is not obvious whether this contribution is equally important to pregnancy success. For example, it is well established that the paternal genome supports growth of extraembryonic tissues whereas the maternal genome fosters development of the embryo proper (Barton et al., 1984). After fertilization, the development of an embryo is controlled by maternal genomic information that is accumulated during oogenesis (Telford et al., 1990). It is only at the 8-cell stage in the bovine embryo that the embryonic genome activates and the embryo switches to transcribing its own RNA (Memili and First, 2000). The parental genomes differentially contribute to the phenotypic diversity of the individual, which has been clearly demonstrated in the aforementioned studies.

Most fertility studies in cattle have focused on cow fertility; therefore, male fertility has not been thoroughly investigated, with few studies reported (Feugang et al., 2009; Khatib et al., 2010; Peñagaricano et al., 2012). Given that most breeding schemes in cattle are focused 
on the selection of elite bulls using progeny testing or genomic selection, and that some semen traits (e.g., sperm motility and percentage of abnormal sperm) show moderate to high heritability (Druet et al., 2009), the characterization of bull fertility markers is feasible and would improve reproductive performance in cattle .

A recent comparative genomics study characterized many genes involved in the control of spermatogenesis that were highly conserved from fly to human (Bonilla and $\mathrm{Xu}, 2008)$. Interestingly, some of these genes were reported to be crucial for human fertility. However, it is not known whether these spermatogenesis genes play important roles in the fertility of bulls. As such, based on the spermatogenesis similarities between flies and humans, we hypothesize that highly conserved spermatogenesis genes are involved in cattle fertility. Therefore, the first objective of this study was to carry out an association analysis between highly conserved spermatogenesis genes and sire conception rate (SCR) as a measure of bull fertility. In a previous study, we reported significant associations of single genes and epistatic interactions from the POU1F1 pathway with female fertility traits (Khatib et al., 2009). Furthermore, the association of some members of the POU1F1 pathway with SCR was observed in a population of 222 Holstein bulls (Khatib et al., 2010). Thus, the second objective of this study was to validate these associations in a different and larger bull population. Significant polymorphisms associated with bull fertility can be used as genetic markers in breeding programs aimed at improving reproductive performance in cattle.

\section{MATERIALS AND METHODS}

Associations of candidate fertility genes examined in this study were carried out in 2 experiments. In the first part, SNP in the spermatogenesis genes and the POU1F1 pathway genes were tested for associations with SCR in a large bull population. In the second part, genes found significantly associated with SCR were tested for association with female fertility traits (fertilization and blastocyst rates). Male fertility genes that play roles in female fertility can be used to improve reproductive performance in cattle using genetic information from both males and females in breeding schemes.

\section{Gene Selection, SNP Identification, and Genotyping for Bull Fertility}

In total, 58 spermatogenesis genes, with conserved testicular expression from fly to human, were reported in Bonilla and $\mathrm{Xu}$ (2008). Of those genes, only 22 were annotated in the bovine genome. For SNP identification and genotyping, genomic DNA was extracted from semen samples of 268 Holstein bulls (Genex Cooperative/ CRI, Shawano, WI) using standard phenol-chloroform protocols. One DNA pool was constructed from 20 random semen samples with equal amounts of DNA. The DNA pool was amplified using primers designed in the 22 candidate genes to amplify $5^{\prime}$ untranslated regions (UTR), exons, introns, and $3^{\prime}$ UTR. The PCR products were sequenced and SNP identified by visually inspecting sequence traces. The PCR amplification and sequencing were performed as described in Khatib et al. (2008). Table 1 shows the primer sets used to amplify the 9 candidate spermatogenesis genes found to be polymorphic in the bull population.

The POU1F1 pathway genes were selected in this study because they have shown significant associations with female and male fertility traits in previous studies (Khatib et al., 2009, 2010). Genotyping of POU1F1

Table 1. Primers used to amplify the 9 spermatogenic genes

\begin{tabular}{|c|c|c|c|c|}
\hline Gene & Accession no. & Forward primer & Reverse primer & $\begin{array}{l}\text { Product } \\
\text { size (bp) }\end{array}$ \\
\hline$D C U N 1 D 1$ & 539869 & ATACCCTTAGGCAGTTAG & AATTGTAAACCCTGAGAC & 536 \\
\hline$D D X_{4}$ & 493725 & AAACACGGAACAGAGGGT & AGGCAGGATTAGCAAGTATG & 404 \\
\hline DNAI1 & 524709 & CGGTAAGTGAGCAGCATC & ACTGAAGCCTTTGCCCTA & 495 \\
\hline DNAI1 & & CCCAGTGCTCCAAATCCT & ATGGCTCATCTTGTCTTCAGTA & 413 \\
\hline DNAI1 & & CGTGACTGGGTTTAGGAT & CTGGTGGCTGCTGTCTAT & 602 \\
\hline GSTM3 & 615507 & TTCTCTTCCCTGCAAGTCGT & TGAGAACAGCTGCCATCATC & 664 \\
\hline$M A P 1 B$ & 514739 & CCATTTCCTAAGGCACAG & TTCCGCCATCTTCCTACA & 504 \\
\hline$M A P 1 B$ & & CTTATGGTCGTGATTATGAA & AAGGCTAACACTGCTGGT & 502 \\
\hline$M A P 1 B$ & & GGCTGTGACATACCTACC & CAGACCTTCCCTACTTATT & 645 \\
\hline PPP1R11 & 504846 & CACATTACGGCGGAACTA & ATCCCAAGCAGTATCACCTA & 591 \\
\hline PPP1R11 & & ACCTGTTCTATCTCCTCCCA & GTCACCTACCCACCTTGC & 543 \\
\hline SPATA20 & 511363 & TTGGAGAAGAAACCCACCAG & CCTCACAAGCAAGGCTAAGG & 459 \\
\hline
\end{tabular}


pathway polymorphic genes (POU1F1, GHR, STAT5A, $O P N$, and $U T M P$ ) was completed as described in Khatib et al. (2009). A total of $29 \mathrm{SNP}-24$ located in the 9 spermatogenic genes and 5 located in the POU1F1 pathway genes - were genotyped in the Genex population (268 animals) by matrix-assisted laser desorption/ ionization-time-of-flight (MALDI-TOF) MS (GeneSeek Inc., Lincoln, NE).

\section{Imputation}

To increase the sample size and improve the statistical power of the study, the 29 SNP identified in the 14 candidate genes and genotyped in 268 bulls (hereafter the reference population) were imputed in a total of 1,720 bulls (hereafter the imputed population) so that a final data set of 1,988 bulls with genotypic data was generated for subsequent statistical analyses. Bulls in the reference and imputed populations have been previously genotyped with the Bovine SNP50 Bead Chip (Illumina Inc., San Diego, CA); hence, shared SNP were used to infer the genotypes of the unshared SNP in the imputed population. Genotypes of the reference $(\mathrm{n}=268)$ and the imputed populations $(\mathrm{n}=1,720)$ for the Bovine SNP50 Bead Chip were provided by Genex Cooperative/CRI and the Animal Improvement Programs Laboratory of the United States Department of Agriculture (AIPL-USDA, Beltsville, MD), respectively. Single nucleotide polymorphisms with minor allele frequencies below $5 \%$ were removed. After data editing, 38,265 SNP spanning the entire bovine genome were available in both populations for the imputation process.

Imputation of SNP was carried out for each candidate gene separately. In each case, 100 SNP on each side of the gene were used to infer the genotypes of the ungenotyped SNP. Imputation was performed using the population-based haplotype clustering algorithm of Scheet and Stephens (2006), which was implemented via fastPHASE software (version 1.2) using the default settings for all parameters (University of Washington TechTransfer Digital Ventures Program, Seattle).

\section{Phenotypic Data for Bull Fertility}

The 1,988 bulls genotyped with the Illumina Bovine SNP50 Bead Chip were evaluated for SCR, a phenotypic evaluation of bull fertility provided to dairy producers by AIPL-USDA, as described in Peñagaricano et al. (2012). Briefly, SCR is the expected difference in conception rate of a sire compared with the mean of all other evaluated sires (Kuhn and Hutchison, 2008; Kuhn et al., 2008). In this study, SCR values ranged from -10.66 to $+6.80 \%$, and the number of breedings per bull ranged from 303 to 111,402. The SCR data were obtained from 7 consecutive evaluations provided by AIPL-USDA between August 2008 and December 2010. For bulls with multiple evaluations, the most recent SCR evaluation was used in the analysis.

\section{Statistical Analysis for Bull Fertility}

The association between each SNP and SCR was evaluated using the following mixed linear model:

$$
\mathrm{SCR}_{i j k l}=\mu+\mathrm{EVAL}_{j}+\beta \mathrm{SNP}_{k}+\operatorname{sire}_{l}+\mathrm{e}_{i j k l},
$$

where $\mathrm{SCR}_{i j k l}$ is the dependent variable, $\mu$ is the general mean, $\mathrm{EVAL}_{j}$ is the fixed effect of the $j$ th AIPL-USDA SCR evaluation $(j=1,2, \ldots, 7), \mathrm{SNP}_{k}$ is the number of copies of one allele of the SNP (corresponding to 0 , 1 , or 2 copies) carried by the $i$ th animal $(i=1,2, \ldots$, 1988), $\beta$ is the regression coefficient for the SNP considered (also known as the allele substitution effect), sire $_{l}$ represents the random additive genetic effect of the $l$ th sire $(l=1,2, \ldots, 246)$ of the $i$ th animal, and $\mathrm{e}_{i j k l}$ represents the random residual for each observation. To detect possible deviations from the additive model, associations between genotype and SCR were evaluated using SNP as a categorical variable.

Random effects were assumed to follow the multivariate normal distribution:

$$
\left(\begin{array}{l}
\mathbf{s} \\
\varepsilon
\end{array} \mid \sigma_{s}^{2}, \sigma_{\varepsilon}^{2}\right) \sim N\left[\mathbf{0},\left(\begin{array}{cc}
\mathbf{A} \sigma_{s}^{2} & \mathbf{0} \\
\mathbf{0} & \mathbf{W}^{-1} \sigma_{\varepsilon}^{2}
\end{array}\right)\right],
$$

where $\mathbf{s}$ and $\varepsilon$ are the vectors of sire and residual effects, respectively; $\sigma_{s}^{2}$ and $\sigma_{\varepsilon}^{2}$ are the sire and residual effect variances, respectively; $\mathbf{A}$ represents the matrix of additive relationships between sires in the pedigree $(1,558 \times 1,558)$, and $\mathbf{W}$ is a diagonal matrix of order 1,988 with its elements representing reliabilities of SCR values. The A matrix was calculated based on a 5-generation pedigree of sires downloaded from AIPL-USDA. The association between each SNP and SCR was tested using a likelihood ratio test by comparing to a reduced model without the SNP effect.

To further evaluate the contribution of the POU1F1 pathway to bull fertility, interactions between adjacent genes in the pathway were tested. For this purpose, a reduced additive model was compared with a full interactive model, and the significance of the interaction term was tested using a likelihood ratio test. 


\section{Phenotypic and Genotypic Data for Cow Fertility}

The most significant SNP for SCR (rs109423562 located in $M A P 1 B$ ) was further investigated for association analysis with fertilization and blastocyst rates - the main cow fertility traits - using an in vitro fertilization (IVF) system. The procedures of IVF and subsequent embryo culture were described in Khatib et al. (2008). To generate fertilization and blastocyst rate data, 6,282 IVF were performed and 4,207 embryos produced using oocytes from 359 ovaries collected from 359 Holstein cows and semen samples from 12 Holstein bulls. For 74 ovaries, oocytes were fertilized by 2 different bulls each. Fertilization rate was calculated as the number of cleaved embryos at d 2 post-fertilization divided by the total number of fertilized oocytes collected from 1 ovary. Blastocyst rate was calculated as the number of embryos that reached the blastocyst stage (d 8) and appeared normal out of the total number of embryos produced.

The 359 ovaries were genotyped for SNP rs109423562 (G/A) using PCR-RFLP. A 171-bp fragment was amplified using the primers 5'-GCAGCTCTTTTAGGAGTGTTAGCGTCTGAT-3' (forward) and 5'-CTCACAGAGGGCATTTGACA-3' (reverse). The PCR product was then digested by the restriction enzyme HinfI and electrophoresed on a 2.0\% agarose gel. Allele $\mathrm{G}$ was cut, whereas allele $\mathrm{A}$ was uncut.

\section{Statistical Analysis for Cow Fertility}

Association between SNP rs109423562 (G/A) in $M A P 1 B$, and fertilization and blastocyst rates were analyzed using the following mixed linear model:

$$
\mathrm{y}_{i j k}=\mu+\operatorname{ovary}_{i}+\operatorname{sire}_{j}+\mathrm{SNP}_{i j k}+\mathrm{e}_{i j k}
$$

where $\mathrm{y}_{i j k}$ represents the fertilization or blastocyst rate of oocyte $k$ from ovary $i$ fertilized with semen from bull $j, \mu$ represents a general mean for the trait considered, ovary $_{i}$ represents the random effect of the individual ovary from which oocytes were harvested, sire ${ }_{j}$ represents the random effect of the sire used in the fertilization, $\mathrm{SNP}_{i j k}$ represents the fixed effect of the ovary genotype for the SNP considered, and $\mathrm{e}_{i j k}$ represents the residuals, assumed normal, independent, and identically distributed with mean 0 and variance $\mathbf{I} \sigma_{e}^{2}$. Ovaries and bulls were assumed uncorrelated, with variance structures $\mathbf{I} \sigma_{o}^{2}$ and $\mathbf{I} \sigma_{s}^{2}$, respectively. Association between the SNP and fertilization or blastocyst rate was tested again using a likelihood ratio test by comparison with a reduced model without the SNP effect. All statistical analyses were performed using the pedigreemm package
(Vazquez et al., 2010) of the R language/environment (R Development Core Team, 2009).

\section{RESULTS}

\section{SNP Identification and Association of Candidate Genes with SCR}

Sequencing analysis revealed $24 \mathrm{SNP}$ in 9 spermatogenesis genes (DCUN1D1, DNAI1, DDX4, GAPDHS, GSTM3, MAP1B, PPP1R11, SPATA20, and UBC) in the bull population using the pooled DNA sequencing approach. These SNP were not identified in the Illumina Bovine SNP50 Bead Chip . Five SNP previously identified in 5 genes from the POU1F1 pathway (Khatib et al., 2009) were also included in this study. Table 2 shows a complete description of these SNP, including minor allele frequency, position in the genome, and location in the gene. Overall, 29 SNP located in 14 candidate genes were tested for association with SCR in a population of 1,988 bulls.

For spermatogenesis genes, $3 \mathrm{SNP}$ located in $M A P 1 B$ and $1 \mathrm{SNP}$ in PPP1R11 showed significant associations with SCR (Table 3). The most significant SNP in $M A P 1 B$ was SNP5.MAP1B, located in intron 5 , with an allele substitution effect of -0.24 and a $P$-value of 0.001. The other 2 significant SNP were SNP1.MAP1B and SNP3.MAP1B, both located in intron 1, with an allele substitution effect of 0.15 and $P$-values of 0.025 and 0.039, respectively. After Bonferroni correction for multiple testing, only SNP5.MAP1B remained significant $(P$-value $=0.024)$. Pairwise linkage disequilibrium (LD) tests showed moderate $\mathrm{LD}\left(\mathrm{R}^{2}=0.38\right)$ between SNP1.MAP1B and SNP3.MAP1B and between SNP1. MAP1B and SNP5.MAP1B. The LD between SNP3. MAP1B and SNP5.MAP1B was relatively low $\left(\mathrm{R}^{2}=\right.$ 0.14). SNP1.PPP1R11 located in the $5^{\prime}$ UTR region of PPP1R11 showed significant association with SCR, with an allele substitution effect of 0.15 and a $P$-value of 0.046 .

For the POU1F1 pathway, individual gene analysis revealed significant associations of POU1F1 and $S T A$ T5A with SCR (Table 3 ). SNP1.POU1F1, a Pro $\rightarrow$ His mutation located in exon 3 of POU1F1, showed an allele substitution effect of -0.20 with a $P$-value of 0.035 . In addition, SNP1.STAT5A - a synonymous mutation located in exon 8 of STAT5A - showed an allele substitution effect of 0.13 and a $P$-value of 0.046. SNP1. OPN, located in $O P N$, did not show association with SCR using SNP as a linear covariate. When using the SNP genotype as a categorical variable in the model, this $O P N$ SNP showed evidence of association with bull fertility $(P=0.052)$ with an overdominance effect (data not shown). However, further studies are required 
Table 2. Description of the 29 SNP used in this study (SNP located in POU1F1 pathway genes are in bold)

\begin{tabular}{|c|c|c|c|c|c|c|c|}
\hline SNP ID & Alleles ${ }^{1}$ & $\mathrm{MAF}^{2}$ & Chromosome & $\begin{array}{l}\text { Position } \\
\text { (UMD3.1) }\end{array}$ & Gene & Location $^{3}$ & dbSNP ID ${ }^{4}$ \\
\hline SNP1.POU1F1 & $\mathrm{C} / \mathrm{A}$ & 0.16 & 1 & 35013926 & POU1F1 & Pro-His & NA \\
\hline SNP1.DCUN1D1 & $\mathrm{C} / \mathrm{T}$ & 0.23 & 1 & 84663386 & $D C U N 1 D 1$ & Intronic & rs41572761 \\
\hline SNP1.GSTM3 & $\mathrm{G} / \mathrm{A}$ & 0.46 & 3 & 33769554 & GSTM3 & Intronic & rs110810231 \\
\hline SNP2.GSTM3 & $\mathrm{G} / \mathrm{A}$ & 0.46 & 3 & 33769863 & GSTM3 & Intronic & rs110543356 \\
\hline SNP1.OPN & $\mathrm{C} / \mathrm{T}$ & 0.42 & 6 & 38122665 & $O P N$ & Intronic & rs110930453 \\
\hline SNP1.DNAI1 & $\mathrm{T} / \mathrm{C}$ & 0.21 & 8 & 77190020 & $D N A I 1$ & Syn & rs109443442 \\
\hline SNP2.DNAI1 & $\mathrm{T} / \mathrm{C}$ & 0.20 & 8 & 77190050 & $D N A I 1$ & Syn & rs110651925 \\
\hline SNP3.DNAI1 & $\mathrm{T} / \mathrm{A}$ & 0.36 & 8 & 77227374 & $D N A I 1$ & Intronic & rs109212402 \\
\hline SNP4.DNAI1 & $\mathrm{C} / \mathrm{T}$ & 0.44 & 8 & 77200698 & $D N A I 1$ & Intronic & rs 43567772 \\
\hline SNP1.UBC & $\mathrm{T} / \mathrm{C}$ & 0.47 & 17 & 53142315 & $U B C$ & Intronic & rs110054817 \\
\hline SNP2.UBC & $\mathrm{T} / \mathrm{C}$ & 0.47 & 17 & 53142330 & $U B C$ & Intronic & rs109440807 \\
\hline SNP1.GAPDHS & $\mathrm{T} / \mathrm{G}$ & 0.35 & 18 & 46389772 & $G A P D H S$ & Intronic & rs109299248 \\
\hline SNP2.GAPDHS & $\mathrm{T} / \mathrm{C}$ & 0.35 & 18 & 46391451 & $G A P D H S$ & Intronic & rs109941190 \\
\hline SNP1.SPATA20 & $\mathrm{A} / \mathrm{G}$ & 0.26 & 19 & 36800176 & SPATA20 & Syn & rs110711008 \\
\hline SNP1.STAT5A & $\mathrm{C} / \mathrm{G}$ & 0.43 & 19 & 43045807 & STAT5A & Syn & rs137182814 \\
\hline SNP1.MAP1B & $\mathrm{T} / \mathrm{C}$ & 0.48 & 20 & 9415997 & $M A P 1 B$ & Intronic & rs110809733 \\
\hline SNP2.MAP1B & $\mathrm{C} / \mathrm{T}$ & 0.18 & 20 & 9415902 & $M A P 1 B$ & Intronic & rs109009656 \\
\hline SNP3.MAP1B & $\mathrm{G} / \mathrm{A}$ & 0.30 & 20 & 9415740 & $M A P 1 B$ & Intronic & rs108965715 \\
\hline SNP4.MAP1B & $\mathrm{G} / \mathrm{A}$ & 0.50 & 20 & 9367286 & $M A P 1 B$ & Intronic & rs109674740 \\
\hline SNP5.MAP1B & $\mathrm{G} / \mathrm{A}$ & 0.26 & 20 & 9331992 & $M A P 1 B$ & Intronic & rs109423562 \\
\hline SNP6.MAP1B & $\mathrm{C} / \mathrm{T}$ & 0.50 & 20 & 9367402 & $M A P 1 B$ & Intronic & NA \\
\hline SNP1.DDX4 & $\mathrm{T} / \mathrm{C}$ & 0.26 & 20 & 23382814 & $D D X_{4}$ & Intronic & NA \\
\hline SNP2.DDX4 & $\mathrm{C} / \mathrm{T}$ & 0.26 & 20 & 23410221 & $D D X_{4}$ & Intronic & NA \\
\hline SNP1.GHR & $\mathrm{T} / \mathrm{A}$ & 0.17 & 20 & 31909478 & $G H R$ & Phe-Tyr & NA \\
\hline SNP1.UTMP & $\mathrm{C} / \mathrm{T}$ & 0.14 & 21 & 59667572 & $U T M P$ & Syn & rs132991801 \\
\hline SNP1.PPP1R11 & $\mathrm{T} / \mathrm{G}$ & 0.27 & 23 & 28710268 & PPP1R11 & $5^{\prime} \mathrm{UTR}$ & rs109300808 \\
\hline SNP2.PPP1R11 & $\mathrm{G} / \mathrm{T}$ & 0.17 & 23 & 28708894 & PPP1R11 & Intronic & NA \\
\hline SNP3.PPP1R11 & $\mathrm{G} / \mathrm{A}$ & 0.19 & 23 & 28708841 & PPP1R11 & Intronic & NA \\
\hline SNP4.PPP1R11 & $\mathrm{A} / \mathrm{T}$ & 0.18 & 23 & 28709168 & PPP1R11 & Intronic & NA \\
\hline
\end{tabular}

${ }^{1}$ Alleles are ordered as major allele/minor allele.

${ }^{2}$ Minor allele frequency.

${ }^{3}$ Synonymous SNP are indicated as Syn whereas nonsynonymous SNP are indicated by the AA coded by the 2 alleles. UTR $=$ untranslated region.

${ }^{4} \mathrm{NA}=$ no dbSNP ID (http://www.ncbi.nlm.nih.gov/snp/).

Table 3. Genetic markers significantly associated with sire conception rate (SCR)

\begin{tabular}{|c|c|c|c|c|}
\hline SNP ID & Gene & $\begin{array}{l}\text { Genotype } \\
\text { (no.) }\end{array}$ & $\begin{array}{l}\text { Allele substitution } \\
\text { effect } \pm \text { SE } \\
(\mathrm{SCR}, \%)\end{array}$ & $P$-value \\
\hline SNP1.POU1F1 & POU1F1 & $\begin{array}{l}\text { CC }(1413) \\
\text { CA }(530) \\
\text { AA }(45)\end{array}$ & $-0.20 \pm 0.01$ & 0.035 \\
\hline SNP1.MAP1B & $M A P 1 B$ & $\begin{array}{l}\text { GG }(463) \\
\text { GA }(987) \\
\text { AA }(538)\end{array}$ & $0.15 \pm 0.01$ & 0.025 \\
\hline SNP3.MAP1B & $M A P 1 B$ & $\begin{array}{l}\text { CC }(972) \\
\text { CT }(847) \\
\text { TT }(169)\end{array}$ & $0.15 \pm 0.01$ & 0.039 \\
\hline SNP5.MAP1B & $M A P 1 B$ & $\begin{array}{l}\text { CC }(1081) \\
\text { TC }(763) \\
\text { TT }(144)\end{array}$ & $-0.24 \pm 0.01$ & 0.001 \\
\hline SNP1.PPP1R11 & PPP1R11 & $\begin{array}{l}\text { TT }(1033) \\
\text { TG }(821) \\
\text { GG }(134)\end{array}$ & $0.15 \pm 0.01$ & 0.046 \\
\hline SNP1.STAT5A & STAT5A & $\begin{array}{l}\text { CC }(656) \\
\text { CG }(967) \\
\text { GG }(365)\end{array}$ & $0.13 \pm 0.01$ & 0.046 \\
\hline
\end{tabular}


to validate the overdominance effect in a larger sample size and in an independent population.

To further characterize the contribution of the entire POU1F1 pathway to bull fertility, genotypic interactions between adjacent genes in the pathway were tested. Genotypes of STAT5A and UTMP showed a significant interaction with a $P$-value of 0.025 . The most remarkable difference in the interaction term was between genotypes CC/GG $(\mathrm{n}=485)$ and $\mathrm{GG} / \mathrm{AA}(\mathrm{n}$ $=12$ ) of STAT5A/UTMP, with a difference of $1.67 \%$ SCR in favor of the CC/GG genotype and a standard error of 0.096 .

\section{Association of MAP1B with Fertilization Rate and Embryo Survival Rate}

SNP5.MAP1B, located in intron 5 of MAP1B, showed the most significant association with SCR in the bull population analyzed above. To characterize its effect on female fertility, we tested the association of this SNP with fertilization rate and blastocyst rate in the IVF system. SNP5.MAP1B showed significant associations with both fertilization rate $(P=0.027)$ and blastocyst rate $(P=0.029$; Table 4$)$. Oocytes collected from genotype $\mathrm{CT}$ cows showed the lowest fertilization rate $(59.9 \%)$ compared with that from $\mathrm{CC}(66.4 \%)$ and TT $(66.3 \%)$ cows (Table 4). For blastocyst rate, the CT genotype again showed the lowest rate (27.1\%), whereas homozygous CC and TT individuals showed blastocyst rates of 31.0 and $41.8 \%$, respectively (Table 4). Interestingly, SNP5.MAP1B showed marked nonadditive effects for both IVF fertility traits.

\section{DISCUSSION}

The objective of this study was to test the hypothesis of association of polymorphisms in conserved spermatogenesis genes and in the POU1F1 pathway genes with bull fertility using a data set of 1,988 bulls. The spermatogenesis genes MAP1B and PPP1R11 and the POU1F1 genes STAT5A and POU1F1 showed significant associations with SCR. Three SNP in MAP1B that were in low to moderate LD were significantly associated with SCR. However, after correction for mul- tiple testing, only 1 SNP in intron 5 showed significant association with SCR.

The $M A P 1 B$ gene belongs to the microtubule-associated protein family and is known to affect neuronal development such as axon growth (Tymanskyj et al., 2012), development of dendritic spine and synaptic maturation (Tortosa et al., 2011), and regulation of the interaction between microtubules and actin microfilaments for axonal development (Montenegro-Venegas et al., 2010). However, recent reports on the expression of $M A P 1 B$ in the male reproductive tract in both rat and human (Queiróz et al., 2006) and in testis of fruit fly and mouse (Bonilla and $\mathrm{Xu}, 2008$ ) suggest important functions of this gene in the regulation of male fertility. Thus, the association with SCR reported in this study supports the role of $M A P 1 B$ in male fertility across a wide range of species.

A SNP in the $5^{\prime}$ UTR of PPP1R11 was associated with SCR in the bull population examined in this study. This is the first report of association between male fertility in cattle and PPP1R11, which is consistent with previous reports on the roles of this gene in spermatogenesis in mouse and human. For example, the different isoforms of PPP1R11 (also known as TCTEX5) were found to be expressed in most mouse tissues with high expression in testis, epididymis, and in the head and tail regions of spermatozoa (Han et al., 2007). In a subsequent study, it was shown that mutations in the long transcript of PPP1R11 were associated with normal sperm function (Han et al., 2008). The authors concluded that PPP1R11 plays important roles in sperm motility and spermatogenesis. A recent study reported that an isoform of protein phosphatase 1 (PP1 $\gamma 2)$, which has an essential role in spermatogenesis, forms a complex with PPP1R11 in the testis (Cheng et al., 2009). Given that PP1 $\gamma 2$ is regulated by PPP1R11, these results further support the idea that PPP1R11 has important functions in spermatogenesis.

The spermatogenesis genes investigated in this study were selected from a pool of genes whose expression is highly conserved in testis of both fruit fly and mouse (Bonilla and $\mathrm{Xu}, 2008$ ). The protein sequence identities between cattle and human and between cattle and fly are 91 and $32 \%$, respectively, for $M A P 1 B$, and 99

Table 4. Association between SNP5 of MAP1B gene and fertilization rate and blastocyst rate

\begin{tabular}{lccccc}
\hline & \multicolumn{2}{c}{ Fertilization rate } & & \multicolumn{2}{c}{ Blastocyst rate } \\
\cline { 2 - 3 } \cline { 5 - 5 } $\begin{array}{l}\text { Genotype } \\
\text { (no.) }\end{array}$ & Estimate $\pm \mathrm{SE}$ & $P$-value & & Estimate $\pm \mathrm{SE}$ & $P$-value \\
\hline CC (321) & $0.664 \pm 0.03$ & 0.027 & & $0.310 \pm 0.02$ & 0.029 \\
CT (84) & $0.599 \pm 0.03$ & & & $0.271 \pm 0.03$ & \\
TT (28) & $0.663 \pm 0.04$ & & & $0.418 \pm 0.05$ & \\
\hline
\end{tabular}


and $47 \%$, respectively, for $P P P 1 R 11$. As such, the association of these genes with bull fertility testifies to the usefulness of the comparative genomics approach in selecting candidate male fertility genes.

Single gene analysis of STAT5A and members of the POU1F1 pathway revealed significant associations of SNP in these genes with SCR using a population of 1,988 bulls. In a previous study, we demonstrated significant associations between some members of the POU1F1 pathway and estimated relative conception rate in a small $(\mathrm{n}=222)$ bull population (Khatib et al., 2010). Thus, results of this study confirm previous reports of the involvement of STAT5A and POU1F1 in male fertility traits. To test whether epistatic interactions affect male fertility, we analyzed associations of genotypic combinations of adjacent genes in the pathway with SCR. Significant genotypic interactions were found for STAT5A and UTMP. Interestingly, genotypic interactions of STAT5A and UTMP were also found to be significant for fertilization and embryo survival rates in an IVF system in cows (Khatib et al., 2009). The IVF system was originally constructed to identify genes associated with cow fertility measured as fertilization success and embryo development (Khatib et al., 2008). Male and female fertility traits are supposedly different because they are products of different organ structures and different physiology. Indeed, embryonic genomic activation and genomic imprinting provide strong evidence that the contribution of the 2 parental genomes to organism survival is not necessarily equal. Nonetheless, fertilization and embryo development require contributions of genetic material of both male and female. Thus, associations of the POU1F1 pathway genes with male and female fertility traits support the notion that these genes need to be contributed by both parents to ensure successful embryo development.

To further explore the involvement of male fertility genes identified in this study in female fertility, we tested the association of the most significant gene, MAP1B, with fertilization and embryo survival rates using data from the IVF system. The MAP1B genotypes of the cows, from which oocytes were extracted and used for fertilization and embryo culture, were significantly associated with differential fertilization rate and embryo survival rate. Interestingly, MAP1B SNP showed nonadditive effects on both fertilization and blastocyst rates. The heterozygotes showed a lower fertilization rate than the homozygotes. Although underdominance is relatively rare, similar effects have reported in previous studies (Lin et al., 1987; Eckert et al., 2009). However, further studies are required to validate these effects. Recently, the expression of $M A P 1 B$ was found to be downregulated in follicular cystic follicles compared with normal follicles, suggesting that alteration in $M A P 1 B$ expression may be involved in reproductive failure in cattle (Choe et al., 2010). These results again demonstrate the significance of both parental genomes to embryonic development and fertility.

\section{CONCLUSIONS}

Although the mechanisms by which the spermatogenesis genes identified in this study regulate bull fertility are not known, the functional conservation of these genes from fly to human, their expression in testis, and their association with female fertility traits provide a strong incentive for further functional analysis. In addition, validation of the association of the POU1F1 pathway genes with male fertility in a large bull population suggests that these genes can be used as genetic makers for reproductive performance in cattle.

\section{ACKNOWLEDGMENTS}

The study was supported by USDA Hatch grant WIS-142-PRJ17PH from the University of WisconsinMadison. The authors thank Genex Cooperative/CRI (Shawano, WI) for providing genotypic and phenotypic data. The authors also thank Ashley Driver, Ricky Monson, and John Parrish (all of University of Wisconsin, Madison) for assistance with in vitro fertilizations and embryo culture.

\section{REFERENCES}

Amann, R. P., and J. M. DeJarnette. 2012. Impact of genomic selection of AI dairy sires on their likely utilization and methods to estimate fertility: A paradigm shift. Theriogenology 77:795-817.

Barton, S. C., M. A. Surani, and M. L. Norris. 1984. Role of paternal and maternal genomes in mouse development. Nature 311:374376.

Bonilla, E., and E. Y. Xu. 2008. Identification and characterization of novel mammalian spermatogenic genes conserved from fly to human. Mol. Hum. Reprod. 14:137-142.

Cheng, L., S. Pilder, A. C. Nairn, S. Ramdas, and S. Vijayaraghavan. 2009. PP1 $\gamma 2$ and PPP1R11 are parts of a multimeric complex in developing testicular germ cells in which their steady state levels are reciprocally related. PLoS ONE 4:e4861.

Choe, C., Y. W. Cho, C. W. Kim, D. S. Son, J. Han, and D. Kang. 2010. Identification of differentially expressed genes in bovine follicular cystic ovaries. Korean J. Physiol. Pharmacol. 14:265-272.

Dobson, H., R. Smith, M. Royal, Ch. Knight, and I. Sheldon. 2007. The high-producing dairy cow and its reproductive performance. Reprod. Domest. Anim. 42(Suppl. 2):17-23.

Druet, T., S. Fritz, E. Sellem, B. Basso, O. Gérard, L. Salas-Cortes, P. Humblot, X. Druart, and A. Eggen. 2009. Estimation of genetic parameters and genome scan for 15 semen characteristics traits of Holstein bulls. J. Anim. Breed. Genet. 126:269-277.

Eckert, A. J., A. D. Bower, J. L. Wegrzyn, B. Pande, K. D. Jermstad, K. V. Krutovsky, J. B. St Clair, and D. B. Neale. 2009. Association genetics of coastal Douglas fir (Pseudotsuga menziesii var. menziesii, Pinaceae). I. Cold-hardiness related traits. Genetics 182:1289-1302.

Feugang, J. M., A. Kaya, G. P. Page, L. Chen, T. Mehta, K. Hirani, L. Nazareth, E. Topper, R. Gibbs, and E. Memili. 2009. Two-stage 
genome-wide association study identifies integrin beta 5 as having potential role in bull fertility. BMC Genomics 10:176.

Han, Y., X. X. Song, H. L. Feng, C. K. Cheung, P. M. Lam, C. C. Wang, and C. J. Haines. 2008. Mutations of t-complex testis expressed gene 5 transcripts in the testis of sterile t-haplotype mutant mouse. Asian J. Androl. 10:219-226.

Han, Y. B., H. L. Feng, C. K. Cheung, P. M. Lam, C. C. Wang, and C. J. Haines. 2007. Expression of a novel T-complex testis expressed 5 (Tctex5) in mouse testis, epididymis, and spermatozoa. Mol. Reprod. Dev. 74:1132-1140.

Khatib, H., W. Huang, X. Wang, A. H. Tran, A. B. Bindrim, V. Schutzkus, R. L. Monson, and B. S. Yandell. 2009. Single gene and gene interaction effects on fertilization and embryonic survival rates in cattle. J. Dairy Sci. 92:2238-2247.

Khatib, H., R. L. Monson, W. Huang, R. Khatib, V. Schutzkus, H. Khateeb, and J. J. Parrish. 2010. Short communication: Validation of in vitro fertility genes in a Holstein bull population. J. Dairy Sci. 93:2244-2249.

Khatib, H., R. L. Monson, V. Schutzkus, D. M. Kohl, G. J. M. Rosa, and J. J. Rutledge. 2008. Mutations in the STAT5A gene are associated with embryonic survival and milk composition in cattle. J. Dairy Sci. 91:784-793.

Kuhn, M. T., and J. L. Hutchison. 2008. Prediction of dairy bull fertility from field data: Use of multiple services and identification and utilization of factors affecting bull fertility. J. Dairy Sci. 91:2481-2492.

Kuhn, M. T., J. L. Hutchison, and H. D. Norman. 2008. Modeling nuisance variables for prediction of service sire fertility. J. Dairy Sci. 91:2823-2835.

Lin, C. Y., A. J. McAllister, K. F. Ng-Kwai-Hang, J. F. Hayes, T. R Batra, A. J. Lee, G. L. Roy, J. A. Vesely, J. M. Wauthy, and K. A. Winter. 1987. Association of milk protein types with growth and reproductive performance of dairy heifers. J. Dairy Sci. 70:29-39.

Memili, E., and N. L. First. 2000. Zygotic and embryonic gene expression in cow: A review of timing and mechanisms of early gene expression as compared with other species. Zygote 8:87-96.

Montenegro-Venegas, C., E. Tortosa, S. Rosso, D. Peretti, F. Bollati, M. Bisbal, I. Jausoro, J. Avila, A. Cáceres, and C. Gonzalez-Billault. 2010. MAP1B regulates axonal development by modulating Rho-GTPase Rac1 activity. Mol. Biol. Cell 21:3518-3528.
Peñagaricano, F., K. A. Weigel, and K. Khatib. 2012. Genome-wide association study identifies candidate markers for bull fertility in Holstein dairy cattle. Anim. Genet. 43:65-71.

Queiróz, D. B., A. M. Silva, G. Gutiérrez-Ospina, C. S. Porto, G. Grossman, P. Petrusz, and M. C. Avellar. 2006. Cells positive for microtubule-associated protein $1 \mathrm{~B}$ (MAP $1 \mathrm{~B}$ ) are present along rat and human efferent ductules and epididymis. Cell Tissue Res. 325:125-133.

R Development Core Team. 2009. R: A Language and Environment for Statistical Computing. R Foundation for Statistical Computing, Vienna, Austria.

Santos, J. E. P., W. W. Thatcher, R. C. Chebel, R. L. A. Cerri, and K. N. Galvao. 2004. The effect of embryonic death rates in cattle on the efficacy of estrus synchronization programs. Anim. Reprod. Sci. 82-83:513-535.

Scheet, P., and M. Stephens. 2006. A fast and flexible statistical model for large-scale population genotype data: Applications to inferring missing genotypes and haplotypic phase. Am. J. Hum. Genet. 78:629-644.

Shook, G. E. 2006. Major advances in determining appropriate selection goals. J. Dairy Sci. 89:1349-1361.

Telford, N. A., A. J. Watson, and G. A. Schultz. 1990. Transition from maternal to embryonic control in early mammalian development: A comparison of several species. Mol. Reprod. Dev. 26:90-100.

Tortosa, E., C. Montenegro-Venegas, M. Benoist, S. Härtel, C. González-Billault, J. A. Esteban, and J. Avila. 2011. Microtubuleassociated protein 1B (MAP1B) is required for dendritic spine development and synaptic maturation. J. Biol. Chem. 286:4063840648.

Tymanskyj, S. R., T. M. Scales, and P. R. Gordon-Weeks. 2012. MAP1B enhances microtubule assembly rates and axon extension rates in developing neurons. Mol. Cell. Neurosci. 49:110-119.

Vazquez, A. I., D. M. Bates, G. J. M. Rosa, D. Gianola, and K. A. Weigel. 2010. Technical note: An R package for fitting generalized linear mixed models in animal breeding. J. Anim. Sci. 88:497-504.

Weigel, K. A. 2006. Prospects for improving reproductive performance through genetic selection. Anim. Reprod. Sci. 96:323-330. 